\title{
HPV and Adolescent Males with HIV 2007-2009
}

Martinez $\mathbf{J}^{1}$, Brakebill $\mathbf{C}^{2}$, Carleton RA ${ }^{3}$, Bansal $\mathbf{A}^{4}$, Malviya $\mathbf{P}^{4}$, Hosek $\mathbf{S}^{5}$, Littlejohn $\mathbf{P}^{6}$, Simples $\mathbf{P}^{7}$ and Henry-Reid LM ${ }^{8}$

${ }^{1}$ Division of Adolescent and Young Adult Medicine, Stroger Hospital of Cook County/Ruth Rothstein CORE Center, Chicago, Illinois, USA

${ }^{2}$ Ruth Rothstein CORE Center, Adolescent Medicine Program at the CORE Center, Chicago, Illinois, USA

${ }^{3}$ Department of Psychology, De Paul University, Chicago, Illinois, USA

${ }^{4}$ Department of Pediatrics, Stroger Hospital of Cook County, Chicago, Illinois, USA

${ }^{5}$ Division of Child and Adolescent Psychiatry, Department of Psychiatry, Stroger Hospital of Cook County, Chicago, Illinois, USA

${ }^{6}$ Ruth Rothstein CORE Center, Pediatrics/ Adolescent Medicine, Chicago, Illinois, USA

${ }^{7}$ Department of Pathology, Stroger Hospital of Cook County, Chicago, Illinois, USA

${ }^{8}$ Division of Adolescent and Young Adult Medicine, Stroger Hospital of Cook County/Ruth Rothstein CORE Center, Chicago, Illinois, USA

\begin{abstract}
Objective: Human Papillomavirus (HPV) infection is found to be more prevalent in HIV-infected than uninfected men $(73 \%$ vs. $43 \%)$. Young men who have sex with men (YMSM) with HIV with HPV anal lesions often deny or are unaware of these lesions. Unless screened, these lesions will remain undiagnosed with a potential for transmission to sexual partners and disease progression. We explored screening practices for HPV and therapeutic/diagnostic outcome of HPV screening among YMSM with HIV, including co-infection with other STIs. Methods: Chart review of all HIV+ adolescent males, age 13-24 years, reporting MSM, engaged in care, 2007-2009, at an inner city HIV clinic for adolescents and young adults.

Results: The average number of males enrolled in care was 143 per year (range 127-166) and none received HPV vaccination. By visual inspection 50 YMSM were identified with anal HPV lesions: $46 \%$ received anal cytology, $30 \%$ were identified as abnormal (ASCUS, LGSIL, or HGSIL); $90 \%$ received medical treatment (Imiquimod or Podophylline); $72.3 \%$ with no evidence that lesions cleared; $68.6 \%$ were referred to Colorectal surgical clinic. In addition, $62 \%$ had STIs diagnosed: syphilis (42\%); gonorrhea (39\%); chlamydia (26\%), and HSV (19\%), Hepatitis B $(6 \%)$; Hepatitis C $(3 \%)$. Clearance of HPV was associated with having more than one STI $(p=0.03)$.

Conclusions: Detecting genital warts by visual inspection without acetic acid is an insensitive screening, thus the number of males suspected to have HPV would be higher than the 50 identified by visual inspection. In this cohort of HIV+ YMSM with HPV, a third were identified as having abnormal cytology, which is worrisome for infection with oncogenic HPV subtypes. Not all received HPV medical and or referred for surgical treatment. The majority of the cohort was co-infected with STIs, and having more than one STI was associated with clinical clearance of HPV lesions.
\end{abstract}

Keywords: Human papillomavirus; HPV; HIV; Genital warts; Condyloma; Adolescents; YMSM; Sexually transmitted infections; Anal cytology

\section{Introduction}

Genital Human Papillomavirus (HPV) infection is one of the most prevalent STIs diagnosed in USA, causing genital warts and is strongly associated with genito-anal dysplasia (also known as squamous intraepithelial lesion (SIL)) [1,2]. Routine reporting of HPV is not yet a recommendation from Centers for Disease Control and Prevention (CDC) [3] and CDC also does not routinely recommend screening for HPV in asymptomatic adolescents, except in young men who have sex with men (YMSM) and in pregnant adolescent females [4]. Prevalence of HPV varies by gender and age: $28 \%-82 \%$ among adolescent girls and young adult women [5,6] and 29\%-48\% among adolescent and young adult males [7-9], with prevalence higher among youth 20 to 24 years old (14.5\% among $14-19$, and $44.8 \%$ among $20-24$ year olds) [10]. A one year prospective study of YMSM in an urban center (mean age $=21$ ) revealed that $70 \%$ had any HPV infection detected by clinician collected anal swab HPV DNA testing [11], although HPV DNA testing is not FDA approved for HPV screening in men. HPV infection in men, contributes to further transmission of HPV infection and subsequent cervical disease in women [12] as well as to HPV infection and subsequent disease (cancers) among YMSM; and accounts for $5.2 \%$ of cancers worldwide $[13,14]$.

Anal HPV is of particular concern in its association with anal cancer. Incidence trends between 1975 and 2008 of anal cancer (anus, anal canal, and anorectum) reveal an increase of $2.2 \%$ annual percent change per year (APC) and a mortality rate (1980-2008) increase of $1.4 \%$ APC [15]. Of note, is that squamous cell anal cancer occurs at much higher rates in HIV-infected MSM (approximately 60-160/100,000) $[16,17]$ compared to the general population $(1 / 100,000)$ [18]. Thus, assessing for presence of infection and implementing treatment may be one method to reduce further transmission of HPV and anal cancer, in addition to the HPV vaccines.

\section{HPV and HIV}

HPV is particularly prevalent among MSM (57\%) [19] with a two fold increase among MSM who are also infected with HIV (93-95\%) [20]. HIV infected MSM in turn have a sixty fold increase in HPV infection compared to men in the general population [21]. Detection

*Corresponding author: Jaime Martinez, Division of Adolescent and Young Adult Medicine, Stroger Hospital of Cook County/Ruth Rothstein CORE Center, Chicago, Illinois, USA, Tel: 312-864-3573; Email: jmartinez@cookcountyhhs.org;jmart335@att.net

Received October 22, 2014; Accepted December 20, 2014; Published December 30, 2014

Citation: Martinez J, Brakebill C, Carleton RA, Bansal A, Malviya P, et al. (2014) HPV and Adolescent Males with HIV 2007-2009. J AIDS Clin Res 6: 409 doi:10.4172/2155-6113.1000409

Copyright: ( 2014 Martinez J, et al. This is an open-access article distributed under the terms of the Creative Commons Attribution License, which permits unrestricted use, distribution, and reproduction in any medium, provided the original author and source are credited. 
is also higher among HIV infected men (73\%) compare to those who are sero negative (43\%) in heterosexual relationships [22]. Anecdotally, adolescent males with HIV, and especially those whose risk factor is MSM, often present with anal HPV lesions, although many deny or unaware of these lesions, and are often missed if the clinician fails to examine the anal and or genital areas.

\section{HPV Detection}

Generally, detecting genital warts on visual inspection accounts for about $1 \%$ of individuals infected. A comparison of methods for assessing HPV infection revealed that in comparison to $1 \%$ on visual inspection noting genital warts, other methods yielded higher numbers of individuals diagnosed with HPV: $4 \%$ were detected by colposcopy, $10 \%$ by HPV DNA (negative on colpo); $60 \%$ by presence of HPV antibodies (who were negative by HPV test); $25 \%$ of individuals screened did not show infection by any method [23,9]. However, there is currently no FDA approved test to detect HPV in men [24]. HPV tests which detect viral nucleic acid or capsid protein, have been approved by the FDA for screening women 30 years old and older, but not for screening men or for women less than 20 years of age [4].

The use of anal-rectal cytology (ARC) is being used for evaluating HPV disease of the anal canal in MSM and especially in those with HIV disease. HPV anal cytology has been demonstrated to have good negative predictive value for assessing HPV disease and found to be useful in follow-up of patients with anal disease [24]. Further, New York State has issued recommendations for screening all HIV infected MSM at baseline and annually by performing anal cytology [26]. To our knowledge no other state has published similar recommendations.

We explored screening practices of medical providers for HPV among HIV infected YMSM. We wanted to understand if screening practices for HPV resulted in treatments and in referrals to Colorectal or Dermatology specialties and if clinicians documented whether treatment(s) made a difference in resolution of lesions.

\section{Method}

A chart review was conducted of all HIV infected adolescent males, age 13-24 years, reporting MSM, engaged in care between 2007-2009, at an inner city HIV clinic for adolescents and young adults. Vaccination for immunizing against human papilloma virus was not available at the clinic and not provided during this time period. Pathology samples by way of biopsy and or anal Papanicolaou test (PAPs) were reviewed, as well as presence of other sexually transmitted infections (STIs). This study was approved by the Internal Review Board of the institution.

\section{Results}

Demographics and HPV detection: The average number of males enrolled in care was 143 per year (range 127-166). Fifty HIV infected males were identified by clinicians as having perianal lesions on visual inspection without the use of acetic acid. Participants of these 50 charts were mostly African American (88\%), had a mean age of 21.3 years at time of review for the study; $92 \%(\mathrm{n}=46)$ reporting MSM activity, $24 \%$ had CD4 counts below 200 and 94\% with viral load (VL) 100,000 (bDNA). Forty-six percent $(n=23)$ received anal cytology, $30 \%(n=7)$ of those with anal cytology were identified as abnormal (ASCUS, LGSIL, or HGSIL). Only 35 (70\%) of sample was reported to be on combined antiretroviral treatment (cART) (Table 1).

HPV treatment and referrals: 90\% $(n=45)$ received medical treatment, with Imiquimod prescribed in $96 \%$ of the young men who received treatment. Podophylin was reported in one case, and liquid

\begin{tabular}{|l|c|}
\hline Variable & $\begin{array}{c}\text { Total=50 } \\
\text { No. (\%) }\end{array}$ \\
\hline Mean age (years) (range) & $23.1(18-\mathbf{2 8})$ \\
\hline Race/ethnicity & $44(88)$ \\
African American & $3(6)$ \\
Latinos & $3(6)$ \\
Caucasian & 0 \\
\hline Other/mixed & $46(92)$ \\
\hline Reported MSM & \\
\hline & \\
\hline CD4 count (cells/mm3) & $9(18)$ \\
\hline 500 & $12(24)$ \\
\hline $350-500$ & $17(34)$ \\
\hline $200-350$ & $12(24)$ \\
\hline$<200$ & \\
\hline VL (copies/ml)(bDNA) & $47(94)$ \\
$>100,000$ & $3(6)$ \\
\hline$<100,000$ & $31(62)$ \\
\hline Diagnosed with STI(s) & $16 / 34(47.1)$ \\
\hline Self Report of 100\% condom use & $35 / 50(70)$ \\
\hline On cARTs & $23(46)$ \\
\hline Anal cytology $\quad$ Abn cytology & $7 / 23(30)$ \\
\hline Referrals to Colorectal or Derm clinic & $34(68.6)$ \\
\hline Medical treatment of HPV (Imiquimod; Podophylin, liquid nitrogen). & $45(90)$ \\
\hline & \\
\hline
\end{tabular}

Table 1: Characteristics of sample of young men with HIV and perianal HPV lesions $(n=50)$.

\begin{tabular}{|l|c|c|c|c|}
\hline Variable & Referred & Not referred & Chi-Square & P value \\
\hline Clinically cleared HPV & $20.6 \%$ & $46.2 \%$ & 3.07 & .08 \\
\hline Adherence to cART $>95 \%$ & $58.6 \%$ & $87.5 \%$ & 2.30 & .13 \\
\hline $100 \%$ condom use & $50.0 \%$ & $45.8 \%$ & 0.05 & .82 \\
\hline Initial CD4 Count & 277 & 281 & $\begin{array}{c}\text { Time } \times \text { Status } \\
\text { interaction: F = .102 }\end{array}$ & .75 \\
\hline
\end{tabular}

Table 2: Associated variables for referral to specialty care (Colorectal or Dermatology clinics).

nitrogen in 3 cases. No cases of Fluorouracil (5FU) were documented. Referrals to Dermatology or Colorectal was noted in $68.6 \%(n=34)$. No reasons for or against referrals were documented in the charts (Table 1).

Clearing or resolution of clinically visible HPV lesions: $27.7 \%$ $(n=13)$ of the sample had evidence documented that the HPV lesions had resolved within the study period of 3 years (2007-2009).

Referred vs. non-referred: Comparing those who received a Colorectal or Dermatology clinic referral $(n=34)$ vs. without referral $(n=13)$ revealed that those who did not receive a referral trended more likely $(46.2 \%$ vs. $20.6 \%)$ to see their HPV clear $(\mathrm{p}=0.08)$. Those in the referred group were less likely (but not significantly) to have cART medication adherence above 95\% (58.6\% for the referral group, $87.5 \%$ for the non-referred). Self-reported condom use of $100 \%$ was higher for the referred group $(50.0 \%$ vs. $45.8 \%)$ ( $p>0.82)$. No relationship was found between referrals and clearing of HPV, adherence to cART, condom use, or CD4 counts (Table 2).

Co-STI infections: Sixty-two percent $(n=31)$ of the 50 young men were also diagnosed with other sexually transmitted infections (STIs). Syphilis was most prevalent ( 13 cases), followed by gonorrhea ( 12 cases), chlamydia (8 cases), and HSV ( 6 cases), along with 2 cases of Hepatitis $\mathrm{B}$, one of Hepatitis $\mathrm{C}$, and one of genital "molluscum contagiosum". Of those with STI co-infections, 55\% $(n=17)$ had multiple STIs diagnosed over the 3 year period of this study (Table 3 ).

Predicting HPV clearance: Binary logistic regressions or chi- 


\begin{tabular}{|l|c|}
\hline STI type present & No. (\%) \\
\hline Syphilis & $13(41.9)$ \\
\hline Gonorrhea & $12(40)$ \\
\hline Chlamydia & $8(27)$ \\
\hline Herpes Simplex Genital Infections & $6(20)$ \\
\hline Hepatitis B & $2(6)$ \\
\hline Hepatitis C & $1(3)$ \\
\hline Molluscum & $1(3)$ \\
\hline$>1$ STI present & $17(55)$ \\
\hline
\end{tabular}

Table 3: Prevalence of STI Type $(n=31)$

\begin{tabular}{|l|c|c|c|}
\hline & Beta & Odds Ratio & P value \\
\hline Initial CD4 count & $(.002)$ & 0.99 & .35 \\
\hline Initial viral load & .000 & 1.00 & .60 \\
\hline Multiple STIs & 1.49 & 4.44 & .03 \\
\hline Abn PAP & $(1.36)$ & 0.26 & .26 \\
\hline$>95 \%$ cART adherence & 1.15 & 3.143 & .20 \\
\hline $100 \%$ condom use (self-report) & .000 & 1.00 & 1.00 \\
\hline
\end{tabular}

Table 4: Binary logistic regressions predicting HPV clearance.

squares, depending on the variable, were performed for the following variables: initial CD4 count, initial viral load, whether there were multiple STIs, whether there had been an abnormal anal cytology, whether the participant had $95 \%$ or better medication adherence to cARTs, and whether there was $100 \%$ condom use, with whether they had any effect on HPV clearance. Analysis revealed that having greater than one STIs demonstrated a greater odds of clearing HPV and was statistically significant $(p=0.3)$. Being $>95 \%$ adherent to cARTs also demonstrated greater odds of clearing HPV but did not demonstrate significance $(\mathrm{p}=0.20)$. Having an abnormal PAP test demonstrated less odds of clearing visible HPV lesions but was not statistically significant $(\mathrm{p}=26)($ Table 4).

Consistent documentation within the charts did not exist for number of present or lifetime partners, gender of partners, circumcision, or smoking history (initiation or cessation).

\section{Discussion}

Detecting genital warts by visual inspection without acetic acid is an insensitive screening method, thus the number of males with HPV would be suspected to be higher than the 50 identified in this study by visual inspection alone. Using visual inspection without the use of acetic acid, anal warts was found among YMSM who on initial enrollment to clinical services had suppressed immune system: $58 \%$ had CD4 counts under 350 (24\% under 200$)$ and $94 \%$ with viral loads $>100,000$. Although $47.1 \%$ reported $100 \%$ condom use, $62 \%$ presented with STIs (and of these, 55\% had more than one STI)-highlighting that they were engaging in high risk sexual behaviors. Presently, there is lack of standardization for HPV screening in men and assessing infection, and this remains an issue for clinicians.

In this group of HIV infected YMSM with HPV, none who received vaccinations for HPV, a third were identified as having abnormal cytology, which is worrisome for infection with oncogenic HPV subtypes. Not all received medical treatment and/ or referral for surgical treatment, and viral subtypes were not assessed. Although cytology was used only on those with visual signs of condyloma, it has been found to be sensitive for detecting HPV infection, but having low sensitivity for detecting HGSIL, compared to high resolution anoscopy [27]. While no standards of care presently exist promoted by CDC or
US Preventive Task Services Force for detection and treatment of HPV in men with anal cytology, reported positive predictive value increase with the use of anal cytology from 38 to $78 \%$ and the negative predictive value increased from $46 \%$ to $79 \%$ in a large study of HIV infected compared to uninfected MSM [28]. Anal cytology has also been found to be cost effective, demonstrating between $\$ 7,800$ to $\$ 16,000$ per year of life saved compared to no screening $[29,30]$. Researchers and clinicians in the HPV field have thus recommended anal cytology for screening of anal HPV and anal intraepithelial neoplasia (AIN), with further evaluation in patients with positive anal cytology using High Resolution Anoscopy (HRA) [31].

HRA requires referrals to clinicians trained in HRA, most often to Dermatology or Colorectal clinics. In this cohort of YMSM, clinicians referred $68.6 \%$ of these young men with no documented criteria for or against said referrals but a trend toward those without resolution of their lesions. Those referred were more likely to have unresolved HPV lesions and more likely to demonstrate non-adherence to cART, despite more likely over reporting $100 \%$ condom use. No statistical significance was demonstrated for these variables when comparing those referred to those not referred.

Envisioning clearance of HPV was of particular interest in this cohort in the pre HPV vaccine era for males. Initial CD4 count, initial viral load, or $100 \%$ condom use had no associated effect on HPV clearance. In contrast having a history of more than one STI increase odds of clearing visual lesions and was statistically significant $(\mathrm{p}=0.03)$. This finding is in contrast to a longitudinal multi-national study (Brazil, Mexico, USA) of 1,159 men age 18-70 years old looking at HPV incidence and clearance (with documented history of STIs) concluded that increase number of partners and advancing age were significantly associated with HPV clearance but not with STIs or circumcision. The authors of this international study believed that the greater the number of sexual partners yielded higher exposure to HPV, yielding increase antibodies that possibly could confer protection against subsequent HPV infections [32,33]. Our findings suggest that increase number of STIs (as a result of unsafe sexual behaviors, e.g., lack of condom use and multiple sexual partners) may also lead to improved immune response to HPV (perhaps by way of exposure to HPV subtypes not visually express resulting in increased HPV antibodies). Further, having an abnormal anal cytology was consistent with persistent HPV infection and resulted in decrease odds of reported HPV lesions having cleared; however was not found to be statistically significant. These associated findings, while interesting, could be due to small sample size not generating enough power to demonstrate significance, but also highlights that factors associated with clearance of HPV are better answered with prospective, longitudinal studies.

There is no currently approved test to detect HPV in men; however, clinicians should routinely screen all HIV infected YMSM by visual inspection of genital and anal areas with acetic acid and anal cytology as the prevalence of infection and consequent anal dysplasia appears to be high. Although CDC does not recommend treatment of subclinical infection, men are important sources of HPV infection in both men and women, and strategies to control viral transmission would thus include screening and should ideally target both genders [34]. Since studies in immune-competent women revealed that HPV infection is transient, $[35,36]$ ideally men should require repeat screening over time.

Limitations of this study need to be considered. The results of this small sample size of primarily African American YMSM cannot be generalizable to other male populations, especially outside of an inner city setting. The data obtained was dependent on documentation 
Citation: Martinez J, Brakebill C, Carleton RA, Bansal A, Malviya P, et al. (2014) HPV and Adolescent Males with HIV 2007-2009. J AIDS Clin Res 6: 409. doi:10.4172/2155-6113.1000409

Page 4 of 4

by clinicians who can be inconsistent in their documentation when treating YMSM living with HIV who present with multiple medical and psychosocial issues. In addition, the binary logistic regression should be interpreted with caution since as a chart review; there is no controlling for confounding variables. Our findings in this study, however, provide essential data for development of improved methods of screening, documented risk factors and physical exams, and documented factors and methods for assessing clearance of HPV infection and or lesions.

Further studies are needed to improve screening methods for HPV in YMSM and for monitoring the impact of the HPV vaccine on prevalence and incidence, especially in this era of HPV vaccines.

\section{References}

1. Ho GY, Burk RD, Klein S, Kadish AS, Chang CJ, et al. (1995) Persistent genital human papillomavirus infection as a risk factor for persistent cervical dysplasia. J Natl Cancer Inst 87: 1365-1371.

2. Frazer IH, Medley G, Crapper RM, Brown TC, Mackay IR (1986) Association between anorectal dysplasia, human papillomavirus, and human immunodeficiency virus infection in homosexual men. Lancet 2: 657-660.

3. Division of STD Prevention (1999) Prevention of Genital HPV Infection and Sequelae: Report of an External Consultants' Meeting. Department of Health and Human Services, Atlanta: Centers for Disease Control and Prevention (CDC): 1-41.

4. Centers for Disease Control and Prevention (2010) Sexually Transmitted Diseases Treatment Guidelines, 2010. MMWR 59: 1-109.

5. Burk RD, Ho GY, Beardsley L, Lempa M, Peters M, et al. (1996) Sexual behavior and partner characteristics are the predominant risk factors for genital human papillomavirus infection in young women. J Infect Dis 174: 679-689.

6. Brown DR, Shew ML, Qadadri B, Neptune N, Vargas M, et al. (2005) A longitudinal study of genital human papillomavirus infection in a cohort of closely followed adolescent women. J Infect Dis 191: 182-192.

7. Kataoka A, Claesson U, Hansson BG, Eriksson M, Lindh E (1991) Human papillomavirus infection of the male diagnosed by Southern-blot hybridization and polymerase chain reaction: comparison between urethra samples and penile biopsy samples. J Med Virol 33: 159-164.

8. Svare El, Kjaer SK, Worm AM, Osterlind A, Meijer CJ, et al. (2002) Risk factors for genital HPV DNA in men resemble those found in women: a study of male attendees at a Danish STD clinic. Sex Transm Infect 78: 215-218.

9. Koutsky L (1997) Epidemiology of genital human papillomavirus infection. Am J Med 102: 3-8.

10. Dunne EF, Unger ER, Sternberg M, McQuillan G, Swan DC, et al. (2007) Prevalence of HPV infection among females in the United States. JAMA 297: 813-819.

11. Glick SN, Feng Q, Popov V, Koutsky LA, Golden MR (2015) High rates of incident and prevalent anal human papillomavirus infection among young men who have sex with men. J Infect Dis 209: 369-376.

12. Giuliano AR (2007) Human papillomavirus vaccination in males. Gynecol Oncol 107: S24-26.

13. Parkin DM, Bray F (2006) Chapter 2: The burden of HPV-related cancers. Vaccine 24 Suppl 3: S3/11-25

14. Parkin DM (2006) The global health burden of infection-associated cancers in the year 2002. Int J Cancer 118: 3030-3044.

15. http://seer.cancer.gov/statfacts/html/anus.html

16. Silverberg MJ, Chao C, Leyden WA, Xu L, Tang B, et al. (2009) HIV infection and the risk of cancers with and without a known infectious cause. AIDS 23: 2337-2345.

17. Crum-Cianflone NF, Hullsiek KH, Marconi VC, Ganesan A, Weintrob A, et al. (2010) Anal cancers among HIV-infected persons: HAART is not slowing rising incidence. AIDS 24: 535-543.

18. Chiao EY, Krown SE, Stier EA, Schrag D (2005) A population-based analysis of temporal trends in the incidence of squamous anal canal cancer in relation to the HIV epidemic. J Acquir Immune Defic Syndr 40: 451-455.
19. Chin-Hong PV, Vittinghoff E, Cranston RD, Buchbinder S, Cohen D, et al (2004) Age-Specific prevalence of anal human papillomavirus infection in HIVnegative sexually active men who have sex with men: the EXPLORE study. J Infect Dis 190: 2070-2076.

20. de Pokomandy A, Rouleau D, Ghattas G, Vézina S, Coté P, et al. (2009) Prevalence, clearance, and incidence of anal human papillomavirus infection in HIV-infected men: the HIPVIRG cohort study. J Infect Dis 199: 965-973.

21. Frisch M, Biggar RJ, Goedert JJ (2000) Human papillomavirus-associated cancers in patients with human immunodeficiency virus infection and acquired immunodeficiency syndrome. J Natl Cancer Inst 92: 1500-1510.

22. Mbulawa ZZ, Coetzee D, Marais DJ, Kamupira M, Zwane E, et al. (2009) Genita human papillomavirus prevalence and human papillomavirus concordance in heterosexual couples are positively associated with human immunodeficiency virus coinfection. J Infect Dis 199: 1514-1524.

23. Koutsky LA, Galloway DA, Holmes KK (1988) Epidemiology of genital human papillomavirus infection. Epidemiol Rev 10: 122-163

24. What Men Need to Know About HPV" 2006.

25. Darrach TM, Winkler TM (2004) The ABCs of anal-rectal cytology. CAP Today.

26. HIV Clinical Resource (2007) Primary care approach to the HIV-infected patient. Preventative Medicine.

27. Salit IE, Lytwyn A, Raboud J, Sano M, Chong S, et al. (2010) The role of cytology (Pap tests) and human papillomavirus testing in anal cancer screening. AIDS 24: $1307-1313$.

28. Palefsky JM, Holly EA, Hogeboom CJ, Berry JM, Jay N, et al. (1997) Anal cytology as a screening tool for anal squamous intraepithelial lesions. J Acquir Immune Defic Syndr Hum Retrovirol 14: 415-422.

29. Oon SF, Winter DC (2010) Perianal condylomas, anal squamous intraepithelia neoplasms and screening: a review of the literature. J Med Screen 17: 44-49.

30. Goldie SJ, Kuntz KM, Weinstein MC, Freedberg KA, Palefsky JM (2000) Costeffectiveness of screening for anal squamous intraepithelial lesions and ana cancer in human immunodeficiency virus-negative homosexual and bisexual men. Am J Med 108: 634-641.

31. Mitra S, Crane L (2012) Diagnosis, treatment, and prevention of anal cancer Curr Infect Dis Rep 14: 61-66.

32. Giuliano AR, Lee JH, Fulp W, Villa LL, Lazcano E, et al. (2011) Incidence and clearance of genital human papillomavirus infection in men (HIM): a cohort study. Lancet 377: 932-940.

33. Giuliano AR, Lazcano E, Villa LL, Flores R, Salmeron J, et al. (2009) Circumcision and sexual behavior: factors independently associated with human papillomavirus detection among men in the HIM study. Int $\mathrm{J}$ Cancer 124: $1251-1257$.

34. Hernandez BY, McDuffie K, Goodman MT (2006) Comparison of Physicianand Self-Collected Genital Specimens for Detection of Human Papillomavirus in Men. J Clin Microbiol 44: 513-517.

35. Evander M, Edlund K, Gustafsson A, Jonsson M, Karlsson R, et al. (1995) Human papillomavirus infection is transient in young women: a populationbased cohort study. J Infect Dis 171: 1026-1030.

36. Ho GY, Bierman R, Beardsley L, Chang CJ, Burk RD (1998) Natural history of cervicovaginal papillomavirus infection in young women. $\mathrm{N}$ Engl J Med 338 : 423-428. 